\title{
Philosophiques
}

\section{L’authenticité : une esquisse de définition}

\section{Claude Romano}

Volume 47, numéro 1, printemps 2020

URI : https://id.erudit.org/iderudit/1070249ar

DOI : https://doi.org/10.7202/1070249ar

Aller au sommaire du numéro

Éditeur(s)

Société de philosophie du Québec

ISSN

0316-2923 (imprimé)

1492-1391 (numérique)

Découvrir la revue

Citer cet article

Romano, C. (2020). L'authenticité : une esquisse de définition. Philosophiques, 47(1), 35-55. https://doi.org/10.7202/1070249ar

\section{Résumé de l'article}

L'objectif de cet article est de cerner la nature de l'idéal d'authenticité, si répandu dans notre culture et nos sociétés contemporaines. Tout d'abord, l'authenticité ne joue-t-elle pas un rôle analogue, par son ampleur et sa vocation à s'appliquer à la totalité de la vie, de ce qu'a été l'idéal de sagesse pour l'Antiquité ? D'autre part, l'authenticité peut-elle se définir simplement comme une sincérité intégrale ? N'est-elle pas plus proche de la vertu d'intégrité ? Nous nous efforçons de montrer l'insuffisance des deux principales voies pour approcher cette notion, celle des théories expressivistes de l'authenticité, d'inspiration romantique, et celle des théories volontaristes qui se sont développées dans le sillage de l'existentialisme : aucune de ces deux approches ne permet de répondre à ce que nous avons appelé le « problème difficile » de l'authenticité.
Ce document est protégé par la loi sur le droit d'auteur. L'utilisation des services d'Érudit (y compris la reproduction) est assujettie à sa politique d'utilisation que vous pouvez consulter en ligne.

https://apropos.erudit.org/fr/usagers/politique-dutilisation/ 


\title{
L'authenticité: une esquisse de définition
}

\author{
CLAUDE ROMANO \\ Université Paris-Sorbonne
}

\begin{abstract}
RÉSUMÉ - L'objectif de cet article est de cerner la nature de l'idéal d'authenticité, si répandu dans notre culture et nos sociétés contemporaines. Tout d'abord, l'authenticité ne joue-t-elle pas un rôle analogue, par son ampleur et sa vocation à s'appliquer à la totalité de la vie, de ce qu'a été l'idéal de sagesse pour l'Antiquité? D'autre part, l'authenticité peut-elle se définir simplement comme une sincérité intégrale? N'est-elle pas plus proche de la vertu d'intégrité? Nous nous efforçons de montrer l'insuffisance des deux principales voies pour approcher cette notion, celle des théories expressivistes de l'authenticité, d'inspiration romantique, et celle des théories volontaristes qui se sont développées dans le sillage de l'existentialisme: aucune de ces deux approches ne permet de répondre à ce que nous avons appelé le «problème difficile» de l'authenticité.
\end{abstract}

ABSTRACT - The aim of this paper is to circumscribe the nature of the ideal of authenticity, so widespread in our culture and our contemporary societies. First of all, does not authenticity play a similar role, by its amplitude and its tendency to be applied to life as a whole, of the ideal of wisdom for Antiquity? On the other hand, is it possible to define authenticity only as a complete sincerity? Is it not closer to the virtue of integrity? This article attempts to show the insufficiency of the two major approaches to that notion: the expressivist conceptions of authenticity, issued from Romanticism, and the voluntarist conceptions that take shape in the wake of Existentialism: neither of these approaches is really able to answer to what we called «the hard problem» of authenticity.

L'idéal d'authenticité personnelle, qu'on s'en réjouisse ou qu'on le déplore, est devenu l'un des piliers de nos sociétés libérales et démocratiques contemporaines, occidentales et non occidentales, comme en témoignent de nombreux phénomènes: le développement des «identités numériques » (Facebook, Twitter), le succès du «développement personnel» et du coaching sous toutes ses formes, l'importation en Occident de spiritualités orientales souvent mal comprises, car subordonnées à l'épanouissement individuel et au culte de la performance ${ }^{1}$, la libération sexuelle, l'affirmation du droit à la différence, l'éclatement des structures traditionnelles du couple et de la famille, et peut-être même, plus près de nous, l'étiolement des structures politiques de l'État-nation — phénomènes très divers, assurément, mais qui ont en commun de placer au centre l'épanouissement de l'individu et la

1. Sur les usages occidentaux du bouddhisme, voir Marion Dapsance, Qu'ont-ils fait $d u$ bouddhisme? Une analyse sans concessions du bouddhisme à l'occidentale, Paris, Gallimard, «Folio essais ", 20I9. 
«réalisation de soi». J'ai essayé de montrer dans Être soi-même $e^{2}$ que ce qu'on peut appeler «les pensées de l'authenticité » (de Rousseau à Larmore en passant par Kierkegaard, Heidegger, Sartre, Taylor) ne représentent, sur le plan historique, que la partie émergée de l'iceberg des pensées de la vérité envers soi-même et d'une vérité qu'il s'agit de faire non en paroles mais "dans sa vie elle-même", comme l'écrit Aristote dans l'Éthique à Nico$m a q u e^{3}$. Cette dernière question remonte en fait aux origines de la philosophie. C'est donc dans la longue durée que je me suis efforcé de réinscrire ces questions en retraçant les prémices de cet idéal typiquement moderne et les différentes formes qu'il a pu prendre, depuis la magnanimité antique, les théories rhétoriques et stylistiques du naturel, jusqu'à l'époque de l'authenticité qui s'ouvre avec Rousseau et le romantisme, et à laquelle nous appartenons encore.

Mon intention n'est pas de revenir sur ces analyses historiques ni sur tous les débats qui entourent cet idéal. Je voudrais plutôt m'interroger dans les réflexions qui suivent sur la spécificité et la cohérence de cette aspiration si répandue à notre époque et chercher à en délimiter les contours. Quel rôle joue l'authenticité dans notre culture, et comment la définir?

\section{L'authenticité - substitut de la sagesse?}

La première hypothèse que je voudrais avancer est la suivante. Il me semble que l'idéal d'authenticité est devenu pour notre époque ce que l'idéal de sagesse a été pour l'époque antique. L'authenticité représente en fait le substitut de la sagesse à une époque qui a cessé de croire en celle-ci. Toute la question est alors de savoir ce qui se joue au juste dans une telle substitution.

L'un des premiers à faire ce diagnostic est un auteur qui a beaucoup réfléchi à ces questions - et qui s'en est aussi beaucoup amusé: Oscar Wilde. En effet, Wilde ne s'est pas contenté de brocarder l'idéal d'authenticité par quelques formules saisissantes — «Soyez vous-même, tous les autres sont déjà pris!» - il a aussi remarqué ce que Foucault et Hadot ont redit beaucoup plus tard, à savoir que l'attrait exercé par l'idéal d'authenticité est d'autant plus puissant dans nos sociétés que cet idéal prend la place laissée vacante par la sagesse. Wilde écrit dans The Soul of Man under Socialism: " "Know thyself" was written over the portal of the antique world. Over the portal of the new world "Be thyself" shall be written ${ }^{4}$."

Qu'est-ce qui se joue dans le passage de "Connais-toi toi-même» à "Sois toi-même"? Dans le monde antique, l'idéal de sagesse était un idéal

2. Cl. Romano, Etre soi-même. Une autre histoire de la philosophie, Paris, Gallimard, coll. «Folio essais», 2018.

3. Aristote, Éthique à Nicomaque, IV, I3, I I 27 a 23-26.

4. O. Wilde, The Soul of Man under Socialism, in Complete Works of Oscar Wilde, Glasgow, Harper-Collins, I994, p. I I79. Une traduction française de ce texte a été publiée sous le titre L'âme humaine, trad. de N. Vallée, Paris, Arléa, 2006. 
d'universalité. Cela signifie d'abord qu'il s'adressait en principe à tout homme - avec naturellement toutes les restrictions nécessaires: en sont exclus les esclaves, les barbares, les enfants et même, dans une large mesure, les femmes, cette universalité pleine et entière ne devenant réellement la règle qu'avec le stoïcisme et son mot d'ordre attribué à Ariston de Chios et traduit en latin par Sénèque sous la forme: sapientia omnis tenet, «la sagesse n'excepte personne ${ }^{5}$. Mais l'universalité de la sagesse doit s'entendre aussi en un second sens, au sens où la sagesse, en dépit des différences que revêt cette notion d'une école à l'autre, consiste à nous élever au-dessus de notre particularité contingente et à atteindre une forme d'universalité: il s'agit de renoncer à nos passions et à nos penchants individuels pour transformer notre vie à l'image du logos impersonnel. Nous devenons pleinement nousmêmes lorsque nous existons sous la conduite de notre noûs, affirme Aristote $^{6}$, ou de notre logos, renchérissent les stoïciens, le façonnement de soi qui aboutit à la sagesse consistant à nous rendre semblable à ce qui nous dépasse - le nồs immortel, le logos divin, l'ordre cosmique, dans le stoïcisme - en faisant taire en nous nos peurs, nos appétits déréglés, nos penchants invétérés, en d'autres termes, en nous débarrassant de notre singularité accidentelle et insignifiante. Pierre Hadot a insisté à juste titre sur cette universalité du sage antique: la sagesse est à la fois modèle universel offert à l'imitation de tout homme et modèle d'universalisation de soi ${ }^{7}$.

C'est pourquoi l'expression «souci de soi» peut facilement prêter à malentendu. Dans l'epimeleia seautou, le souci porte sur soi, si l'on veut, puisque c'est soi-même qu'il s'agit d'élever à la sagesse; mais ce souci ne prend nullement ce «soi» pour fin, si l'on veut dire par là que l'horizon de la préoccupation serait soi-même, sa propre individualité. C'est exactement le contraire qui est vrai: il s'agit de se détourner de soi, de se défaire de soi, de transcender sa propre particularité contingente. La sagesse est le produit d'un «dépassement de soi », comme le rappelle à juste titre Hadot, et non d'un souci de soi entendu comme «un rapport de soi à soi » ${ }^{8}$. Plotin invite par exemple son lecteur à "sculpter sa propre statue ${ }^{9}$ ", mais cette image veut dire chez lui le contraire de ce qu'elle semble vouloir dire pour nos

5. Sénèque, Lettres à Lucilius, 94, 16.

6. C'est en ce sens qu'il faut entendre la formule: «N'est-ce pas avouer que l'intellect (nô̂s), c'est nous-mêmes?" (Aristote, Éthique à Nicomaque, IX, 8, I I69 a I).

7. P. Hadot, Exercices spirituels et philosophie antique, Paris, Albin Michel, 2002.

8. Ibid., p. 326.

9. Plotin, Ennéades, I, 6, 9: «Si tu ne vois pas encore ta propre beauté, fais comme le sculpteur d'une statue qui doit devenir belle: il enlève ceci, il gratte cela, il rend tel endroit lisse, il nettoie tel autre jusqu'à ce qu'il fasse apparaître le beau visage dans la statue. De la même manière, toi aussi, enlève tout ce qui est superflu, redresse ce qui est oblique, purifiant tout ce qui est ténébreux pour le rendre brillant, et ne cesse de sculpter ta propre statue jusqu'à ce que brille en toi la clarté divine de la vertu» (trad. P. Hadot). Voir le commentaire d'Hadot dans Exercices spirituels et philosophie antique, p. 58-59. 
oreilles contemporaines: il ne s'agit pas de sculpter une statue à sa propre image, mais de faire apparaître dans le matériau dont chaque homme est constitué, par un travail de soustraction, l'effigie de la divinité. Lorsque Foucault interprète le souci de soi comme un souci $d u$ soi, et ajoute que le soi est à la fois «sujet du souci, objet du souci ${ }^{10}$ »; quand il fait de ce «soi » «la fin du souci de soi ${ }^{11}$ ", en sorte que cultiver ce souci reviendrait, pour les philosophes hellénistiques, à se prendre soi-même comme fin, à se cultiver soi-même, à s'améliorer soi-même; ou encore, lorsqu'il parle d'une "autofinalisation du rapport à soi ${ }^{12}$ » en vertu de laquelle «le but de la pratique de soi, c'est le soi ${ }^{13}{ }^{\text {", }}$, Foucault n'est pas loin d'un contresens pur et simple, car il fait coïncider les deux formules de Wilde, appartenant à deux constellations philosophiques différentes. Dans le souci de soi antique, «le soi » est, si l'on veut, le point de départ, le matériau, il n'est en aucun cas la fin: nulle autotélie, ici, nulle «auto-finalisation du rapport à soi».

Le gnôti seauton de l'Apollon pythique signifie en réalité l'opposé d'une connaissance de soi en tant qu'individu particulier, de la connaissance de nous-même à laquelle nous invitent les manuels de "développement personnel ». Comme cela a été remarqué depuis longtemps, il doit s'interpréter en relation étroite avec le mêden agan ( "Rien de trop ») et fait signe vers une prise de conscience de nos propres limites en tant qu'hommes en général, y compris les limites de notre possibilité de nous connaître. Se connaître en ce sens-là, comme le souligne Jean Defradas, "c'est surtout connaître sa nature d'homme et ne pas désirer ce qui n'appartient qu'à la divinité ${ }^{14}$ »; c'est donc échapper à l'hubris et cesser de prétendre s'élever au-dessus de la condition humaine. Comme le souligne Pindare dans ses Dialogues pythiques, cette maxime est "pour les mortels, un rappel de leur nature et de leur faiblesse (tôi thnêtôi tês peri auton phuseôs kai astheneias $)^{15}$ ". Il est par conséquent évident que la formule du monde moderne, "Be thyself», veut dire en un sens exactement le contraire de ce que signifiait la formule du monde antique, "Know thyself». L'idéal d'authenticité subvertit l'idéal de sagesse puisqu'il place l'individu au centre de l'attention, et que le genre de vérité qu'il nous prescrit de reconnaître est une vérité située à l'intérieur de nous, et non au-dessus de nous. Elle n'est pas une vérité universelle, mais une vérité particulière. Comme l'écrit le poète anglais Robert Browning,

10. M. Foucault, L'herménentique du sujet. Cours au collège de France (1981-1982), Paris, Gallimard-Seuil-EHESS, 200I, p. 552.

11. Ibid., p. 8I.

12. Ibid., p. I 22 .

13. Ibid.

14. Jean Defradas, Les thèmes de la propagande delphique, Paris, Klincksieck, I954, p. 277.

15. Plutarque, "Sur l'E de Delphes », 394 b, in Dialogues pythiques, Robert Flacelière (dir.), Euvres morales, tome VI, Paris, Les Belles Lettres, I974. 
Truth is within ourselves; it takes no rise

From outward things, what'er you may believe. ${ }^{16}$

Sculpter sa propre statue à l'effigie de cette vérité-là signifie donc quelque chose d'entièrement différent de l'idéal antique de sagesse. Est-ce à dire qu'il n'y a aucun lien entre la formule gravée au frontispice du monde moderne et celle sculptée à l'entrée du monde antique? En un sens, c'est exactement le contraire, puisque, dans les deux cas, il s'agit bel et bien de réaliser une vérité dans sa vie elle-même, ou de se rendre soi-même conforme à la vérité - d'exister en adéquation avec notre être, qu'il soit générique ou individuel. L'idéal d'authenticité représente l'infléchissement moderne, au sein de sociétés individualistes, de l'idéal antique. Foucault a raison, en ce sens, d'affirmer qu'il existe une proximité étroite entre ce qu'il appelle - dans des termes il est vrai anachroniques pour parler de la philosophie hellénistique une "esthétique de l'existence " propre à notre époque, c'est-à-dire une tentative pour façonner notre vie sur le modèle d'une œuvre d'art, et l'idéal antique de la culture de soi. Ce qui est plus étonnant est que Foucault ne semble pas toujours mesurer l'écart qui existe entre ces deux idéaux.

Cet écart, Hegel, en était tout à fait conscient. Le gnôti seauton, affirmait-il dans l'Encyclopédie, ne signifie pour les Anciens qu' "une connaissance de soi selon les aptitudes, le caractère, les inclinations et les faiblesses ", c'est-à-dire un savoir de soi en troisième personne dans lequel l'individu n'occupe par rapport à lui-même aucune position privilégiée. C'est pourquoi, ajoutait-il, cette formule passe à côté de «la signification de la connaissance de ce qu'il y a de vrai dans l'homme, ainsi que de ce qu'il y a de vrai en et pour soi - de l'essence elle-même en tant qu'esprit ${ }^{17}{ }^{\prime}$. Tandis que la formule antique insiste sur des caractéristiques générales, sur les traits qui définissent l'individu en le rapportant à d'autres, la formule moderne met l'accent, de son côté, sur les traits qui définissent l'individu purement en luimême (ce que Hegel appelle l'«esprit»).

Admettons que l'idéal de sagesse et l'idéal d'authenticité se situent à la fois dans la plus grande proximité l'un par rapport à l'autre et dans la plus grande distance. Qu'en résulte-t-il pour la compréhension de l'authenticité ? Une des conséquences qui me paraissent s'ensuivre est «l'ampleur » que possède cet idéal. Contrairement à ce qu'ont soutenu différents auteurs, en effet, par exemple Charles Larmore, il n'est probablement pas possible de concevoir l'authenticité comme une exigence morale parmi d'autres ${ }^{18}$, ni,

16. The Complete Works of Robert Browing, Boston et New York, Houghton, Muffin and co, I899, p. 43.

17. G. W. F. Hegel, Encyclopédie des sciences philosophiques, t. III, Philosophie de l'Esprit, trad. B. Bourgeois, Paris, Vrin, I988, $\$ 377$.

18. Ch. Larmore, Les pratiques $d u$ moi, Paris, PUF, 2004, p. 206: "Mais justement, l'authenticité n'est pas tout, voilà l'erreur fondamentale. Elle n'est qu'une valeur parmi d'autres et il devrait être évident maintenant combien on se trompe en élevant l'authenticité au rang 
comme l'a suggéré Lionel Trilling, de l'appréhender comme une simple transformation de la sincérité, laquelle, de vertu sociale, deviendrait une vertu individuelle. La sincérité représente bien une vertu morale parmi d'autres, et donc une vertu qui peut aisément entrer en conflit avec d'autres vertus - par exemple le tact, la délicatesse, la bienveillance, l'humanité. La sincérité a nécessairement des limites qui lui sont prescrites par la place qu'elle occupe dans l'édifice moral: une sincérité qui n'aurait aucune borne entrerait inévitablement en contradiction avec d'autres exigences éthiques et, par voie de conséquence, avec elle-même. Or ce qui vaut de la sincérité ne vaut pas nécessairement de l'authenticité - et c'est pourquoi celle-ci ne se réduit pas à une sincérité envers soi-même. L'un des traits qui caractérisent les conceptions modernes de l'authenticité, à partir de Rousseau et jusqu'à Heidegger et Sartre, est précisément que l'authenticité y joue le rôle d'idéal suprême, à l'instar de la sagesse dans l'Antiquité, qu'elle ne concerne pas l'existence seulement sous un rapport déterminé mais en tant que tout. L'authenticité concerne l'ensemble de nos conduites et de nos manières d'être, à la manière dont la sagesse antique est censée transformer la vie en totalité. Il serait certes absurde de soutenir que tous les actes d'un être exemplifiant cette qualité sont authentiques - pas plus que tous les actes d'un être courageux ne sont courageux. Comme toute qualité morale, l'authenticité dépend de circonstances dans lesquelles elle peut s'exercer: je ne peux pas être authentique en faisant la queue pour acheter une place de cinéma. D'autre part, comme tout idéal moral, la possession de cette qualité admet des degrés. Il n'en reste pas moins que l'authenticité semble devoir s'étendre à l'ensemble de la vie. Elle ne s'applique pas caeteris paribus, en sorte que l'on pourrait être authentique dans certaines circonstances et inauthentique dans d'autres, comme il convient parfois de dire la vérité, et parfois de la taire. Et cela - telle est du moins mon hypothèse - , parce que l'authenticité se tient dans la plus grande proximité avec l'idéal de sagesse. Rousseau, l'un de ses principaux défenseurs, l'exprime par l'intermédiaire de M. de Wolmar dans la Nouvelle Héloïse, énonçant ainsi ce qui deviendra un leitmotiv des pensées de l'authenticité dans leur ensemble: «Un seul précepte de morale peut tenir lieu de tous les autres, c'est celui-ci: ne fais ni ne dis jamais rien que tu ne veuilles que tout le monde voie et entende ${ }^{19}$.»

Il semble par conséquent difficile de soutenir, à l'instar de Larmore, qu'en certaines circonstances il faudrait être authentique, et dans d'autres non. L'authenticité n'est pas la sincérité. Il semble parfaitement "authen-

d'une valeur suprême [...]. Il convient de dire tout bonnement: nous faisons souvent bien d'être comme un autre. " Et Larmore adopte alors un " "pluralisme éthique" que la tradition philosophique a toujours eu du mal à accepter" (ibid., p. 2I3), pluralisme selon lequel l'authenticité n'est qu'une valeur parmi d'autres. Il conclut que «l'idée d'une “éthique de l'authenticité" [...] repose sur un contresens" (ibid.).

19. J.-J. Rousseau, Julie ou la Nouvelle Hélö̈se, IVe partie, lettre VI; in Euvres complètes, t. II, Paris, Gallimard, «Bibl. de la Pléiade», I96I, p. 424 (nous soulignons). 
tique ", pour un médecin, de taire la maladie dont son patient est affecté ou même de lui mentir à ce sujet s'il pense que la révélation de son état entrầnerait une aggravation de son mal; il semble tout aussi " authentique » de ne pas faire observer à la personne en face de laquelle on est attablé qu'elle vient de se tacher avec son potage. Il ne semble pas contradictoire avec le fait d'être authentique d'avoir des secrets, une vie privée, de ne pas faire étalage de sa vie entière - à cet égard, l'image qu'emploie M. de Wolmar dans ce même passage de la Nouvelle Hélö̈se, celle d'une vie entièrement transparente, offerte au regard de tous, constitue plutôt une caricature de l'idéal d'authenticité qui a probablement ses sources dans le stoïcisme. Le passage cité se poursuit en effet ainsi: "[N]e fais ni ne dis jamais rien que tu ne veuilles que tout le monde voie et entende; et, pour moi, j’ai toujours regardé comme le plus estimable des hommes ce Romain qui voulait que sa maison fût construite de manière qu'on vît tout ce qui s'y faisait ». Womar-Rousseau se fait ici l'interprète de Marc Aurèle (et l'on retrouve par là l'idéal de sagesse): "Seras-tu donc jamais, ô mon âme, bonne, droite, une, nue (agathêe kai aplê kai mia kai gumnê), plus manifeste (phanerôteros) que le corps qui t'enveloppe ?20"

On assiste davantage à une caricature de l'idéal d'authenticité qu'à une expression acceptable de ce qu'il pourrait être. L'authenticité n'est pas une sincérité intégrale, dépourvue de toute limite, et aboutissant à une transparence totale. Pourtant, l'authenticité — comme du reste certaines notions qui la préfigurent, telle la grâce à la Renaissance, lieu d'adéquation parfaite avec soi-même, ou le naturel qui prend forme avec Montaigne et les moralistes classiques - est bel et bien une qualité qui doit s'étendre à la totalité de la vie, devenir une disposition permanente. $\mathrm{Y}$ a-t-il ici contradiction entre ces deux affirmations?

\section{Définir l'authenticité?}

L'authenticité ne semble pas pouvoir être comprise comme une sincérité intégrale ou comme une sincérité qui devrait s'étendre à la moindre péripétie de la vie. Il y a des circonstances dans lesquelles il est légitime de taire la vérité, de la dissimuler et même, peut-être, de la travestir. Le juste qui ment pour sauver des enfants juifs n'est évidemment pas blâmable, sa conduite est courageuse et même admirable. Faire de la sincérité une vertu située audessus de toutes les autres, c'est faire de la vérité de nos énoncés un fétiche, comme si elle devrait l'emporter sur toute autre considération. Sauver la vie d'un innocent ne saurait être mis en balance avec proférer un mensonge. Il est difficile, à cet égard, de souscrire à la thèse de Trilling selon laquelle l'authenticité serait une sincérité absolutisée - même si l'on ne peut nier que Rousseau semble parfois embrasser une telle conception. Comme le 
souligne Montaigne - pourtant un ardent défenseur d'une forme d'authenticité personnelle qu'il désigne du nom de «naturel» et qui est placée au cœur de la démarche littéraire des Essais -, il n'y a aucune contradiction entre faire l'apologie de l'authenticité et admettre que «la vérité mesme n'a pas ce privilege d'estre employée à toute heure et en toute sorte; son usage, tout noble qu'il est a ses circonscriptions et limites ${ }^{21}{ }^{»}$.

Il faut même aller plus loin: le juste qui profère un mensonge dans une situation de violence morale exercée contre lui et contre ceux qu'il entend sauver satisfait à l'idéal d'authenticité au moment où il décide de mentir. On pourrait en effet définir l'authenticité personnelle comme le fait de se conduire et de se présenter soi-même dans sa vie d'une manière qui soit fidèle à la fois à ses principes, aux valeurs auxquelles on souscrit, aux idéaux auxquels on se soumet, et à ses sentiments, inclinations, désirs, préférences, croyances véritables. En la circonstance, le juste se comporte en conformité avec ce qu'il croit et avec les principes éthiques qui guident sa vie. Il estime qu'un mensonge proféré sous la menace est justifié quand il y va de la vie d'innocents, et il se conduit en conséquence. L'authenticité peut être considérée à cet égard comme un concept proche de celui d'intégrité. Est intègre, pourrait-on dire, celui qui règle sa vie et ses conduites sur ses principes éthiques. Pourtant, une différence importante sépare l'intégrité de l'authenticité: l'intégrité est un concept strictement éthique, dans la mesure où les principes sur lesquels s'aligne l'individu intègre touchent à la question du bien et du mal. L'authenticité a affaire à un domaine plus vaste que celui de l'éthique, puisqu'elle embrasse à la fois le faire de vivre selon ses principes et le fait de vivre conformément à ses goûts, à ses aspirations en général, le fait de développer ses propres dispositions et talents; en d'autres termes, il s'agit de «faire la vérité » dans sa vie ou de mener une vie de vérité par rapport à la totalité de ce que l'on est, et pas seulement par rapport à ses principes éthiques. Cela entraîne la conséquence à première vue paradoxale selon laquelle une vie pourrait être authentique même lorsqu'elle déroge aux principes qui régissent le bien-vivre en commun. Et en effet, il existe toute une tendance au sein des pensées de l'authenticité à mettre en exergue des figures d'authenticité rebelles et contestataires, qui s'étend du neveu de Rameau dans le dialogue éponyme de Diderot au Saint Genet de Sartre, en passant par le surhomme nietzschéen et le héraut de La désobéissance civile de Thoreau, une tendance qui tend à faire de la marginalité, de la contestation, quelquefois de l'asocialité et même du crime, des figures d'authenticité. L'authenticité, à la différence de l'intégrité, est éthiquement neutre; elle a même été considérée par certains comme compatible avec l'amoralité. J'y reviendrai plus loin.

On le voit, l'authenticité n'est ni la sincérité ni l'intégrité: elle ressemble à la sincérité en ce sens qu'elle a affaire à la vérité, et consiste à faire

21. M. de Montaigne, Essais, III, XIII, éd. Villey-Saulnier, Paris, PUF, 2004, I078 b. 
la vérité sur soi, à la fois pour soi-même et pour les autres. Tandis que la sincérité consiste simplement à dire ce qu'on pense, et la fiabilité à faire ce qu'on dit, l'authenticité consiste à être ce qu'on est et à se présenter aux autres comme tel. C'est pourquoi une seconde différence importante entre la sincérité et l'authenticité est que celui qui est sincère peut parfaitement se duper lui-même ou être dans l'illusion sur soi - il dit ce qu'il pense ou ce qu'il éprouve, mais il se trompe à ce sujet. Être sincère n'implique aucunement que ce que l'on dit avec sincérité soit la vérité (il suffit que cela soit ce qu'on croit être la vérité). Au contraire, faire la vérité dans sa vie, exister en plein accord avec soi-même, c'est-à-dire avec ce que l'on croit, espère, désire, avec les idéaux auxquels on souscrit, implique nécessairement ne pas être dupe de soi-même, de posséder une certaine lucidité à l'égard de soi. D'autre part, l'authenticité ressemble à l'intégrité puisqu'elle consiste à mener une existence qui soit conforme à ses propres principes, mais elle s'étend au-delà de l'intégrité à des aspects de nous-mêmes qui n'ont pas de statut éthique, comme l'épanouissement de nos propres talents, le fait de suivre nos véritables préférences au lieu de se conformer aux goûts des autres, ou d'écouter nos sentiments véritables au lieu de les confondre avec ceux de notre entourage. L'intégrité consiste à se tenir fermement à ses principes éthiques et à se conduire en conséquence; l'authenticité consiste plutôt en deux attitudes étroitement imbriquées : I) vivre en conformité avec ses principes mais aussi avec ses aspirations en général, ses goûts, ses préférences, ses talents, ses réactions affectives; 2) se présenter aux autres d'une manière qui soit fidèle à ces mêmes principes, aspirations ou réactions affectives, et ne pas chercher à les travestir. L'intégrité est une fidélité à des valeurs qui sont justes ou bonnes, mais pour ainsi dire "impersonnelles »; l'authenticité est une fidélité à ces mêmes principes, mais également à des valeurs plus "personnelles » - désirs, goûts, aspirations pour autant qu'ils sont les nôtres, qu'ils constituent notre propre identité.

Historiquement, si on le considère à partir de Rousseau, on pourrait dire que cet idéal a été conçu selon deux grands modèles que l'on peut baptiser respectivement «expressiviste» et "constructiviste». Le premier, qui est celui du romantisme, met l'accent sur un noyau d'originalité absolue qui résiderait en chaque individu et s'exprimerait en premier lieu dans ses réactions affectives: être authentique, ce serait alors se mettre à l'écoute de la "voix de la nature " qui parle à l'intérieur de chaque homme, du sentiment intérieur qui ne trompe jamais (et ce sentiment intérieur, ou cet "instinct divin ", comme l'appelle Rousseau, représente à maints égards une sécularisation du «témoignage intérieur de la conscience» de Calvin, c'est-à-dire de la certitude qui est gravée dans le cœur de l'élu de son élection, certitude qui, au milieu des doutes et des épreuves, ne chancelle pas). Selon ce premier modèle, par conséquent, la vie de vérité consiste à exprimer dans sa vie sa nature particulière, ce que les théoriciens de cette première lignée de penseurs n'hésitent pas à appeler souvent son «moi». La vie tout entière doit 
devenir alors expression intégrale de celui que l'on est, et l'épicentre de l'être de chacun réside avant tout dans ce qu'il ressent: «Ah, ce que je sais, tout le monde peut le savoir, s'exclame le Jeune Werther; mais mon cœur n'est qu'à $\mathrm{moi}^{22}$.»

Le second modèle pour penser l'authenticité qui s'est imposé avec les « existentialismes» (en un sens très vaste du terme, qui inclut Heidegger) fait reposer au contraire la conformité de l'individu à son être propre dans la fidélité à ses engagements ou à ses choix, voire dans le fait, pour lui, de se "choisir lui-même »; l'épicentre de l'être de chacun réside alors plutôt dans sa volonté. Ce modèle volontariste de l'authenticité a tendance à reposer sur l'idée selon laquelle être authentique équivaudrait à être pleinement résolu ou à exister en conformité avec ses résolutions déjà prises - à exister de manière fidèle à ses propres engagements. On trouve cette idée exprimée chez Kierkegaard à propos du stade éthique, et sous une forme plutôt ironique (puisque le stade éthique est loin de représenter pour le penseur danois, le fin mot d'une existence accomplie), lorsqu'il fait dire à l'assesseur Wilhelm dans L'alternative: "En se choisissant, la personnalité se choisit selon l'éthique, et elle exclut absolument l'esthétique, mais l'homme se choisit pourtant lui-même, sans revêtir par là une autre nature: il devient lui-même (bliver sig selv), et toute l'esthétique retourne à sa relativité23.» On est tout proche, dans ce "choix du choix", comme l'assesseur l'appelle aussi, baptême de la volonté qui fait entrer celle-ci dans le stade éthique, de l'autoposition du moi fichtéen qui s'arrache au néant et se pose lui-même par sa seule puissance discrétionnaire. On retrouve cette même idée d'un "choisir le choix (Wählen der Wabl) ${ }^{24}$ » qui équivaut à la résolution ou à l'être résolu (Entschlossenheit), dans Sein und Zeit, et cette résolution par laquelle le Dasein décide de sa propre existence et de lui-même en totalité est formellement identique à l'authenticité (Eigentlichkeit). "La reprise de soi hors du On, écrit Heidegger, autrement dit la modification existentielle du On-même en être-soi-même authentique doit nécessairement s'accomplir comme re-saisie d'un choix. Mais ressaisir un choix signifie choisir ce choix, se décider pour un pouvoir-être puisé dans le soi-même le plus propre. C'est dans le choix du choix que le Dasein se rend pour la première fois possible son pouvoir-être authentique ${ }^{25}$.» C'est donc avec l'être résolu, et lui seul, qu'est atteinte «la vérité la plus originaire, parce qu'authentique, du Dasein $^{26} »$.

22. Goethe, Les souffrances du jeune Werther, trad. de B. Groethuysen, in Romans, Paris, Gallimard, Bibl. de la Pléiade, I954, p. 7I.

23. Søren Kierkegaard, L'alternative, in Euvres complètes, tome IV, trad. de Paul-Henri Tisseau et Else-Marie Jacquet-Tisseau, Paris, L’Orante, I970, p. I62.

24. M. Heidegger, Sein und Zeit, Tübingen, Max Niemeyer, I6e éd. I986, p. 268; trad. d'E. Martineau, Paris, Authentica, I985, p. I95.

25. Ibid., p. 268 ; trad. citée, p. 195.

26. Ibid., p. 297 ; trad. citée, p. 2 I 2. 
Autre déclinaison de ce motif de l'authenticité comme conformité à un choix fondamental, la théorie sartrienne de l'authenticité, seulement indiquée en creux dans une note de L'être et le néant ${ }^{27}$, et davantage développée dans les Cahiers pour une morale, soutient que l'authenticité réside, pour le Pour-soi, dans l'assomption sans restriction de sa liberté, c'est-à-dire dans un «choix originel» de lui-même et toutes les implications qui découlent de ce choix. L'authenticité consiste alors à vouloir être soi-même et à se vouloir soi-même tel que l'on s'est choisi. À la différence de la «réflexion complice» dans laquelle le Pour-soi se change en En-soi, la «réflexion pure» et authentique est celle dans laquelle le Pour-soi ne s'identifie à rien de donné et se ressaisit à partir de sa pure autoposition (ou autocréation) dans et par son choix: «La réflexion pure et authentique est un vouloir de ce que je veux. C'est le refus de me définir par ce que je suis (Ego) mais par ce que je veux (c'est-à-dire par mon entreprise elle-même non pas en tant qu'elle apparaît à autrui - objectif - mais en tant qu'elle tourne vers moi sa face subjective) [...]. Ainsi la saisie de soi authentique n'est pas du type de l'être, c'est une volonté dirigée sur une volonté2 ${ }^{28}$.»

La particularité de toutes ces conceptions est qu'elles refusent de penser l'authenticité comme conformité à quelque chose de donné, à un ensemble de tendances, de tropismes affectifs ou de dispositions enracinées dans quelque chose comme une "nature", un "caractère" ou un "moi». Sartre va jusqu'à affirmer qu'il n'existe rien à quoi nous puissions être conformes, et que l'idée de conformité à soi ou à son être (ce qu'il appelle "sincérité" dans L'être et le néant) relève purement et simplement de la mauvaise foi. Mais Heidegger, lui aussi, cherche à formuler l'authenticité sans nul recours à une nature de l'individu. Ces conceptions rompent ainsi avec l'approche expressiviste et romantique de l'authenticité. C'est le cas également de Charles Larmore, héritier à plus d'un titre de Sartre, qui estime qu'il est possible de définir l'authenticité simplement en termes de fidélité à ses propres engagements (engagements extérieurs, telle une promesse, ou intérieurs, puisque les états mentaux, tout comme les croyances et les désirs, sont tenus par Larmore pour des engagements). Qu'est-ce alors que l'authenticité? Elle consiste à coïncider avec le principe de notre être qui est d'être engagés à l'égard de nous-mêmes et de nos raisons comme nous seuls pouvons le faire; nous décidons alors de celui que nous avons à être, nous nous façonnons ou nous créons nous-mêmes en tant que nous nous alignons sur nos propres raisons de pensée et d'action. "L'incertitude à laquelle nous mettons [alors] un terme est de nature pratique, non théorique, car il s'agit, dans ce cas, non de découvrir qui nous sommes, mais plutôt de nous

27. Jean-Paul Sartre, L'être et le néant, Paris, Gallimard, I943, rééd. "Tel», 20I3, p. 106.

28. J.-P. Sartre, Cahiers pour une morale, Paris, Gallimard, I983, p. 496. 
façonner ${ }^{29}$. Il s'agit alors, comme l'écrit Larmore en reprenant une formule toute sartrienne, non de coïncider avec un être donné, mais de coïncider «avec notre propre non-coïncidence constitutive ${ }^{30}$ — avec ce que Sartre appelait «liberté» et "choix originel», et que Larmore rebaptise plus modestement nos «engagements".

Chacun de ces modèles, le modèle expressiviste et le modèle volontariste de l'authenticité, soulèvent de sérieuses difficultés.

Commençons par le modèle volontariste. À bien des égards, il constitue une réduction à l'absurde de l'idée même d'authenticité. En effet, s'il n'y a rien à quoi nous puissions nous conformer, et que nous puissions réaliser dans notre vie - aucun ensemble de tendances, de dispositions, de prédilections individuelles —, si notre être est entièrement "construit» par nousmêmes, voire "choisi" en un choix originel, si nous ne sommes que nos choix ("notre être est choix", écrit Sartre) ou, à la rigueur, pour essayer de minimiser ce qu'a d'irréaliste une telle conception, si nous ne sommes qu'un ensemble d' "engagements" que nous prenons nous-mêmes devant nousmêmes, il est difficile de voir comment l'idée de vérité peut encore conserver un sens, puisque la vérité suppose un état de choses indépendant de nous, de ce que nous souhaitons ou croyons. Cet hyper-constructivisme moral qui repose sur un rejet de l'idée même de nature individuelle - et par "nature ", nous entendons ici simplement un ensemble de dispositions et de tendances soustraites à notre volonté et à nos choix, tendances qui peuvent se modifier au cours du temps et n'ont rien d'immuable - ne permet plus vraiment de comprendre, lorsque nous sommes fidèles à nous-mêmes, à quo $i$ au juste nous sommes censés être fidèles. Car si «le moi », comme l'affirme Larmore, se réduit à ses engagements, et si ces engagements auraient toujours pu être autres qu'ils ne le sont, nous avons perdu toute idée d'engagements «justes ", au sens d'appropriés à nous-mêmes, et d'engagements qui ne le sont pas. Si être authentique consiste à être fidèle à ses engagements quels que soient ces engagements, nous nous sommes ôté le moyen de rendre compte du fait indéniable qu'il est tout à fait possible de s'engager à quelque chose (pensée, action) sans que cela reflète le moins du monde ce que nous sommes - simplement parce que nous sommes les jouets de notre entourage ou d'une fausse idée de nous-mêmes. Or toute pensée de l'authenticité doit se donner les moyens de distinguer des engagements faussés, inadéquats, c'est-à-dire procédant d'une forme d'étrangeté à soi ou d'aliénation, et des engagements conformes à notre être - et cet être ne saurait se ramener à une somme d'engagements. On peut dire la même chose du "choix» dont parle Sartre: le fait de choisir en personne quelque chose n'entraîne pas nécessairement que ce que nous avons choisi reflète ce que nous croyons et désirons vraiment - peut-être nous sommes-nous trompés là-dessus. Le même genre de

29. Ch. Larmore, Les pratiques du moi, op. cit., p. I 52.

30. Ibid., p. І9г. 
perplexité vaut pour la résolution heideggérienne. Qu'est-ce qui me prouve, lorsque j'existe selon le mode de la résolution, que ce à quoi je me résous n'est pas l'effet d'une influence du «On» sur moi, et le fait de me vouloir moi-même ou de me choisir moi-même, le refuge du plus parfait conformisme? En d'autres termes, les conceptions volontaristes de l'authenticité semblent s'ôter tout critère de distinction entre des engagements en vertu desquels nous existons en adéquation à nous-mêmes, et des engagements où ce n'est pas le cas, des choix conformes à notre être et des choix qui ne le sont pas, des «résolutions» qui nous arrachent à la voix du «On» et des résolutions qui en sont simplement l'expression. Elles aboutissent à vider d'une bonne partie de son sens l'idée même d'authenticité, c'est-à-dire de vérité ou de conformité à notre être - laquelle suppose que l'être auquel nous nous conformons soit au moins pour une part indépendant de ce que nous voulons ou choisissons.

Pour le dire autrement, de telles conceptions ont tendance à contourner ce qu'a de réellement difficile le problème de l'authenticité, en cherchant à se passer complètement de l'idée d'un discernement de nos véritables tendances, dispositions, désirs, etc., ou d'une découverte de ceux-ci. Or l'idée d'une vérité à l'égard de soi-même présuppose la possibilité d'une telle découverte. Être fidèle à ses engagements ou à ses choix ne suffit pas à être authentique, attendu que je peux parfaitement être dans la plus complète obscurité sur mes véritables désirs au moment où je prends une décision, être totalement aveuglé sur moi-même et étranger à mon être, et celui auquel je serai alors fidèle se réduira à une construction ou à une chimère. En d'autres termes, on pourrait soutenir - aussi paradoxal que cela paraisse - que ces pensées de l'authenticité qui, à maints égards, apparaissent comme les paradigmes de ce type de conception, esquivent purement et simplement ce qu'on pourrait appeler le "problème difficile » de l'authenticité, lequel réside dans le fait qu'il ne suffit pas de s'engager ou de se résoudre à quelque chose pour que ce à quoi on s'engage et se résout soit en adéquation avec notre être véritable: la possibilité de l'aveuglement complet sur soi reste toujours ouverte. Il faut donc un critère additionnel pour distinguer des engagements qui sont conformes à notre être d'autres dans lesquels nous nous aveuglons nous-mêmes sur ce que nous croyons ou voulons vraiment - et un tel critère fait défaut aux conceptions volontaristes.

Quant au paradigme expressiviste, il semble à première vue meilleur dans la mesure où il reconnaît, en deçà de nos choix et de nos engagements, l'existence d'un ensemble de tendances, de dispositions et de tropismes que nous n'avons ni voulus ni choisis, et qui sont constitutifs de notre être singulier. Toutefois, contrairement à ce à quoi on pourrait s'attendre, ce paradigme ne fait pas vraiment droit, lui non plus, au "problème difficile» de l'authenticité. L'idée d' "exprimer» ne fait sens, en effet, que si les sentiments, désirs, croyances que l'on exprime sont véritablement les nôtres. Dans le cas contraire, si nous n'éprouvons pas de peur par exemple, on ne 
dira pas que nous exprimons de la peur, mais que nous jonons la peur ou que nous la simulons. En d'autres termes, il n'y a pas de place, dans le concept d'expression, pour l'idée d'une expression insincère, c'est-à-dire de l'expression d'un sentiment que l'on n'éprouve pas. Bien sûr, il existe un autre concept d'expression, proprement linguistique, selon lequel il est possible de parler de l'expression d'une croyance que l'on n'a pas ou d'un sentiment que l'on n'éprouve pas - mais ce concept n'est pas celui qui est ici en jeu puisque l'expression, au sens du paradigme expressiviste, doit inclure l'expression linguistique et non linguistique, volontaire et involontaire. Par conséquent, une conception qui soutiendrait qu'être authentique, c'est simplement exprimer dans sa vie ses propres dispositions, inclinations, opinions, demeurerait, elle aussi, en deçà du "problème difficile » de l'authenticité, qui porte sur le fait que nous pouvons tout à fait manifester des inclinations qui se révèlent à l'examen n'être pas réellement les nôtres ou être dans l'illusion sur ce que nous désirons vraiment. Tout le roman contemporain, depuis La Princesse de Clèves, ne parle pas d'autre chose que de ce genre de méprise. Le problème de l'authenticité, en d'autres termes, ne peut se réduire à une affaire d'expression, il doit mettre en jeu un discernement et une découverte. Et de cela, le paradigme expressiviste ne semble pas pouvoir davantage rendre compte que le paradigme volontariste.

En outre, il y a une critique qu'il faut adresser conjointement à ces deux paradigmes: ils demeurent prisonniers d'une conception foncièrement individualiste de l'authenticité. Le choix de soi-même, la résolution, l'engagement tel que le conçoit Larmore restent des actes "privés", accomplis en notre for intérieur; quant à l'expression de notre "vrai moi », elle repose souvent, dans la pensée romantique et post-romantique, sur la supposition de l'existence d'un noyau d'originalité irréductible, situé en deçà de toute influence sociale, de toute convention, de toute culture. Un tel noyau d'originalité n'est rien d'autre qu'un mythe. En réalité, notre originalité personnelle naît et se développe toujours au point de jonction de nos tendances particulières et d'innombrables influences sociales. Mais le fait que cette supposition - telle qu'elle sous-tend, par exemple, les conceptions romantiques du génie - soit un mythe ne signifie absolument pas qu'il n'existe pas, pour chacun de nous, un ensemble de tendances et de dispositions relativement stables qui prennent forme peu à peu et sont situées en deçà de nos décisions - une idée que les «existentialismes» semblent avoir été beaucoup trop prompts à rejeter. Il n'y a rien de contradictoire dans une telle idée, ni rien qui contredise notre expérience - au contraire. Il suffit d'être attentif au développement d'un être humain pour constater que celui-ci manifeste très tôt une personnalité propre, distincte de celle des autres membres de sa famille. C'est plutôt l'idée d'un être entièrement autofaçonné, auto-posé et auto-institué par le seul exercice d'une volonté discrétionnaire, ou d'une liberté soustraite à tout ancrage dans des dispositions qui la précèdent, qui est une véritable absurdité. 
Si nous voulons donc donner un sens à l'idée d'authenticité, il faut d'une part admettre une nature individuelle de chacun, située en deçà de tout choix et de toute volonté, et qu'il est possible d'apprendre à discerner pour trouver son orientation dans l'existence, et, d'autre part, admettre que cette "nature " (il n'y a aucune raison de refuser ce terme) se modifie au gré de l'histoire individuelle de chaque être et sous l'effet d'innombrables influences, ce qui ne la disqualifie aucunement pour sous-tendre l'exercice de notre authenticité. En réalité, l'authenticité ne consiste pas seulement en une présentation de soi aux autres qui soit fiable et véridique; elle est ce qui contribue, en vertu de la manière dont nous nous présentons aux autres, à la constitution même de notre identité, au fait pour nous de jouir d'une identité stable que nous puissions endosser devant autrui. L'authenticité sert à «construire » notre identité en tant qu'identité fondamentalement sociale. Elle est le socle d'une dynamique sociale de constitution de soi en vertu de laquelle seul peut avoir une identité au sens ici pertinent celui qui est capable de l'assumer de manière fiable devant les autres - et de l'endosser de cette manière pour qu'elle soit précisément son identité. L'authenticité, au même titre que la sincérité (dire ce qu'on pense) ou la fiabilité (faire ce qu'on dit), a un rôle essentiel à jouer dans la constitution de nos identités, et pas seulement dans leur communication, en sorte que tout ce processus est un processus essentiellement social, et non pas social de manière simplement accidentelle.

\section{Quelques critiques de l'authenticité et quelques réponses}

Cette caractérisation de l'authenticité reste une simple esquisse, mais elle nous permet d'envisager un certain nombre de soupçons et d'objections, issus de différents horizons, qui ont pu être formulés à l'encontre de cet idéal. Je laisserai de côté l'objection issue du "nietzschéisme à la française », comme l'appelle Putnam, selon laquelle la distinction même entre vérité et fausseté serait une illusion (une «erreur»!). Elle est autoréfutante et a donné lieu déjà à une ample littérature. Je laisserai également de côté le reproche issu des cercles les plus conservateurs selon lequel l'idéal d'épanouissement individuel ne serait que l'expression d'une «culture du narcissisme » et d'une forme moderne d'égotisme ou d'égoïsme. En effet, si l'authenticité, comme je l'ai soutenu, consiste à se présenter soi-même et à se conduire dans sa vie d'une manière qui soit fidèle à la fois à ses principes, aux valeurs auxquelles on souscrit, aux idéaux que l'on embrasse, et à ses sentiments, inclinations, désirs, préférences ou croyances véritables, il n'y a strictement aucune raison de supposer que ces désirs, croyances, inclinations sont nécessairement égoïstes, ou ces principes et idéaux, hédonistes, voire nihilistes. Charles Taylor a amplement souligné ce point ${ }^{31}$. L'authenticité, si on la sépare de

31. Charles Taylor, The Ethics of Authenticity, Cambridge, Mass., Harvard University Press, I99I ; trad. de Ch. Melançon, Le malaise de la modernité, Paris, Éditions du Cerf, 20 I 5. 
certaines formulations malheureuses qui ont pu en être données, constitue un idéal exigeant, celui de ne pas transiger avec ses vrais désirs, convictions et principes, et elle peut même conduire à une vie de sacrifices.

Il reste trois objections plus sérieuses que je voudrais examiner.

I) Une critique issue plutôt des sciences humaines et sociales consistant à dénoncer l'élitisme implicite de cet idéal (Bourdieu) ou son inconsistance (Foucault).

2) L'objection selon laquelle l'allégeance à l'idéal d'authenticité conduirait à une forme d'amoralisme.

3) Enfin, l'objection selon laquelle l'authenticité reposerait sur une transparence à soi totale, une connaissance de soi totale qui nous restent inaccessibles.

Du point de vue d'une critique issue des sciences sociales, l'authenticité serait un idéal sournoisement élitiste et bourgeois. Dans L'ontologie politique de Martin Heidegger, Bourdieu considère par exemple que l'Eigentlichkeit heideggérienne est "une retraduction particulière et particulièrement subtile de l'opposition commune entre "l'élite" et les "masses" [...]. Il faudrait recenser, tout au long de ces passages mille fois commentés, les lieux communs de l'aristocratisme universitaire ${ }^{32}$ ". Cette critique englobe d'ailleurs l'inégalitarisme ontologique de Sartre et s'applique à la quête d'authenticité en général: "Égaux en liberté, les hommes sont inégaux dans la capacité à user authentiquement de leur liberté et seule une "élite" peut s'approprier les possibilités universellement offertes d'accéder à la liberté de "l'élite". Ce volontarisme éthique - que Sartre poussera à la limite convertit la dualité objective des destins sociaux en une dualité des rapports à l'existence $[\ldots]^{33}$.»

Or, s'il est clair que l'idéal d'authenticité, depuis ses origines lointaines dans la magnanimité antique, est fortement teinté de connotations aristocratiques - il relève d'un "perfectionnisme moral » qui n'a pu être élaboré et mis en œuvre que par les couches les plus privilégiées de la société -, cela n'entraîne pas qu'il ne puisse être conçu comme à la portée de tous; et c'est en un sens exactement ce qui s'est produit dans nos sociétés occidentales à partir des années soixante: l'authenticité s'est alors démocratisée, elle est devenue même un idéal de masse. On pourrait par conséquent répondre à Bourdieu en lui retournant sa question: le mépris affiché par l'intellectuel pour un idéal qui s'est largement diffusé dans nos sociétés démocratiques contemporaines n'enveloppe-t-il pas une posture largement aussi élitiste que celle qu'il dénonce?

32. P. Bourdieu, L'ontologie politique de Martin Heidegger, Paris, éd. de Minuit, I988, p. 9 I.

33. Ibid., p. 99-100. 
Qu'en est-il de ceux qui, à l'instar de Foucault, ont vu dans cette aspiration collective une revendication totalement creuse et insignifiante? On peut lire par exemple, dans L'herméneutique du sujet, le passage suivant:

En tout cas ce que je voudrais vous signaler, c'est tout de même que quand on voit aujourd'hui la signification, ou plutôt l'absence quasi totale de signification, qu'on donne à des expressions, pourtant très familières et qui ne cessent de parcourir notre discours, comme: revenir à soi, se libérer, être soi-même, être authentique, etc., quand on voit l'absence de signification et de pensée qu'il y a dans chacune de ces expressions aujourd'hui employées, je crois qu'il n'y a pas à être bien fier des efforts que l'on fait maintenant pour reconstituer une éthique du soi ${ }^{34}$.

La posture condescendante adoptée ici par Foucault est d'autant plus surprenante que la conception qu'il défend par ailleurs d'une "esthétique de l'existence» (que pourrait résumer le mot d'ordre "faire de nous-mêmes une œuvre d'art» $)^{35}$ puise sa source, qu'il l'admette ou non, dans l'essor de ces mêmes théories de l'authenticité qu'il fustige. En réalité, Foucault soupçonne la recherche d'authenticité qui s'exprime à son époque d'être la forme contemporaine que revêtent les pratiques ascétiques qui ont vu le jour au sein du christianisme, les techniques de confession et d'aveu que Foucault décrit dans Les aveux de la chair. Foucault oppose à ces techniques aliénantes et assujettissantes visant à arracher aux fidèles ou aux hommes d'Église la vérité sur eux-mêmes aux "techniques de soi» de l'Antiquité, supposées être plus neutres. L'idéal d'authenticité serait un simple avatar des formes traditionnelles d'obéissance telles qu'elles se développent à travers les modalités de la confession chrétienne. Ainsi, de manière assez étrange, ce qui est vécu comme "libération" et "émancipation" par les acteurs euxmêmes (mai 68) est tenu par Foucault pour un discours de sujétion et d'oppression. C'est le cas de la révolution sexuelle elle-même qui ne serait qu'un sous-produit des technologies de contrôle propres aux formes de pouvoir modernes.

Deux choses au moins semblent problématiques dans ce type de position. D'abord, le genre d'attitude qu'adopte Foucault semble réfuté par la pratique. Qui aujourd'hui pourrait élever ses enfants sans faire intervenir l'idéal d'épanouissement de soi? Qui pourrait attendre d'eux qu'ils se bornent à occuper la place qui leur est assignée par la société ? Mais surtout, si l'on suivait à la lettre Foucault, il faudrait conclure que ce sont des techniques de pouvoir qui ont "créé » l'aspiration moderne à connaître ses propres désirs et aspirations et à les réaliser dans sa vie. Est-ce vraisemblable? Comment les techniques de pouvoir pourraient-elles être à la fois répressives, c'est-à-dire empêcher l'épanouissement de nos propres désirs et

34. M. Foucault, L'herméneutique du sujet, op. cit., p. 24I.

35. M. Foucault, Dits et écrits II, Paris, Gallimard, "Quarto", 200I, p. I2 I I. 
aspirations, et être à l'origine de l'aspiration de second degré, propre à l'authenticité, à exprimer et à réaliser ces mêmes désirs et aspirations de premier degré dans notre vie? Une telle conception semble purement et simplement contradictoire.

La seconde objection porte sur la tendance à un relativisme moral, voire à un amoralisme, qui pourrait résulter du passage au premier plan de l'idéal d'authenticité au sein de nos sociétés et de notre culture. On l'a dit, le contraste sur lequel reposent la plupart des conceptions de cet idéal à partir de Rousseau est celui d'une société de fausseté et d'une vérité située à l'intérieur de l'individu. Il n'y a pas à s'étonner, dans ces conditions, que l'individu authentique puisse se revendiquer tel en s'élevant contre le "conformisme» ambiant, et en incluant parfois dans ce conformisme les règles de la morale elles-mêmes. C'est ce qui a favorisé l'émergence des figures de l'authenticité "contestataires" dont il était question plus haut. Pourquoi en effet ne pourrait-on pas fonder sa vie sur un rejet des règles de la morale et de la société, et demeurer malgré tout authentique? Songeons par exemple à la figure de Vautrin dans La Comédie humaine. Vautrin est un malfaiteur qui n'éprouve aucun scrupule à mentir, trahir ou tromper les autres. Mais Vautrin est aussi pourvu d'une grande lucidité sur lui-même et sur les motifs qui le guident. Qu'on relise par exemple le «sermon» qu'il adresse à Lucien de Rubempré à la fin des Illusions perdues, lorsqu'il recueille le jeune homme dans sa voiture au moment où celui-ci est sur le point de se jeter du haut d'un pont, et déguisé en prêtre, lui explique à quel point il a été naiff de croire qu'il pourrait triompher dans le monde au moyen de sa seule bonne foi. Ici, la diatribe du criminel, calquée à maints égards sur le cynisme d'un Gracián et l'immoralisme d'un Nietzsche, consiste à affirmer qu'il faut user de la vérité comme d'un subterfuge et à s'excepter soi-même de la «morale du troupeau». Vautrin est parfaitement sincère avec luimême, et, de surcroît, parfaitement honnête avec son interlocuteur. Il ne cherche pas à s'abuser lui-même ni, au moins en cette circonstance, à abuser quiconque. Il est, pourrait-on dire, à la fois authentique et parfaitement immoral, conformément à sa devise: "Tous les grands hommes sont des monstres $^{36}$."Si l'idéal d'authenticité est compatible avec l'immoralité, cela ne le disqualifie-t-il pas en tant qu'idéal?

Il s'agit d'un problème difficile, mais il me semble qu'on peut lui apporter au moins une esquisse de solution. L'attitude de Vautrin est une possibilité-limite qui convient à un personnage de fiction mais est plus difficile à réaliser dans la vie: cette attitude consiste à dissocier entièrement vérité envers soi-même et fausseté envers les autres. Mais jusqu'à quel point une telle dissociation est-elle possible? Dans quelle mesure ce que l'on pourrait appeler une "hypocrisie pure» est-elle tenable? Examinons un second

36. Honoré de Balzac, Les Illusions perdues, in La Comédie humaine, tome V, Paris, Gallimard, Bibl. de la Pléiade, I977, p. 696. 
personnage, Julien Sorel dans Le Rouge et le Noir. Julien, lui aussi, se déclare au début du roman un "hypocrite consommé ${ }^{37}$ ». Son modèle, avec Napoléon, est Tartuffe. Mais son hypocrisie est plutôt une attitude réactive, un mécanisme de défense, le moyen d'échapper à un père brutal et dépourvu d'amour, à la vie étriquée de petit artisan de province qui l'attend. Ce que va manifester tout le roman, c'est que Julien n'est qu'un hypocrite malgré lui, et que ce à quoi il aspire en réalité, c'est au naturel, la grande qualité stendhalienne, dont Mme de Rênal lui offre le modèle, et Mathilde de la Mole le contre-modèle. À la fin du roman, lorsqu'il meurt sur l'échafaud, il a fini par reconnaître son amour pour la première et a compris que son attrait pour la seconde lui était dicté par l'amour-propre, n'était qu'un "amour de tête » et non un "amour de cœur", selon la terminologie de Stendhal. Il agit alors enfin en plein accord avec lui-même. À propos de son exécution, le narrateur affirme: "Tout se passa simplement, convenablement, et de sa part sans aucune affectation ${ }^{38}$.»

Julien Sorel révèle mieux que Vautrin la véritable nature de l'hypocrisie - celle qui est répandue et commune et dont l'autre n'est qu'une idéalisation. On ne parvient à se revêtir d'un masque de manière convaincante (Julien, quant à lui, ne cesse de se trahir) qu'en adhérant à son propre personnage, c'est-à-dire en finissant par adopter les mêmes désirs et croyances que celui-ci. Le mensonge aux autres, pour pouvoir être soutenu à long terme, exige de la part de celui qui le pratique qu'il se moule sur l'être chimérique auquel il veut donner consistance. Et donc, en réalité, l'hypocrisie envers les autres se double toujours d'un mensonge à soi et d'une perte de repères quant à son identité véritable. Même le criminel le plus cynique est toujours vrai à certains moments au moins pour quelques-uns, et si ce n'était pas le cas, il cesserait de posséder la moindre physionomie morale. C'est ce qui tend à se produire dans des cas extrêmes, comme celui de Jean-Claude Romand tel que le décrit Emmanuel Carrère dans L'adversaire: ici, l'ampleur du mensonge et sa durée conduisent au bord de la dépersonnalisation ${ }^{39}$.

L'authenticité, pour être psychologiquement et existentiellement possible, doit être toujours à la fois une vérité envers soi et envers les autres, car il ne s'agit au fond que des deux faces d'une même monnaie: nous construi-

37. Stendhal, Le Rouge et le Noir, éd. d'Henri Martineau, in Romans, tome I, Paris, Gallimard, "Bibliothèque de la Pléiade", I952, p. 383.

38. Ibid., p. 804 .

39. Emmanuel Carrère cite un rapport psychiatrique: "Il lui sera à tout jamais impossible d'être perçu comme authentique et lui-même a peur de ne jamais savoir s'il l'est. Avant, on croyait tout ce qu'il disait, maintenant on ne croit plus rien et lui-même ne sait que croire, car il n'a pas accès à sa propre vérité et la reconstitue à l'aide des interprétations que lui tendent les psychiatres, le juge, les médias " (L’adversaire, Paris, POL, 2000, rééd. Gallimard, "Folio ", 20I4, p. I 84). Dans l'un de leurs derniers échanges épistolaires, Emmanuel Carrère relève "ce défaut d'accès à vous-même, ce blanc qui n'a cessé de grandir à la place de celui qui en vous doit dire "je" " (ibid., p. 204). 
sons notre identité de manière sociale, en prenant position de manière véridique devant autrui, et c'est en prenant ainsi position que nous découvrons qui nous sommes vraiment. Inversement, nous avons généralement besoin de nous tromper nous-mêmes pour pouvoir tromper les autres, et de tromper les autres pour parvenir à nous faire croire à nous-mêmes ce que nous voudrions croire. Il s'ensuit qu'une authenticité qui coexisterait avec une parfaite hypocrisie n'est qu'un cas d'école dépourvu de plausibilité existentielle. Du reste, même Vautrin éprouve le besoin de se confier à Lucien, ce qui manifeste que son hypocrisie n'est ni "pure» ni universelle; un criminel se cherche des complices, c'est-à-dire précisément des gens qui sont dans la confidence. La possibilité d'une authenticité «immorale» ou "amorale», fondée sur une parfaite hypocrisie, c'est-à-dire sur une duperie systématique des autres eu égard à sa propre identité, n'est pas autre chose qu'une chimère. L'authenticité, si on la pense jusqu'au bout, est difficilement conciliable avec un amoralisme, car on se demande bien comment quelqu'un qui n'aurait aucun principe pourrait assez respecter la vérité pour se la dire à lui-même. Le respect pour la vérité exige plus que sa simple utilité pratique.

Mais l'authenticité est-elle possible? Ne devrait-elle pas nécessairement reposer sur une connaissance de soi et une lucidité à l'égard de soi si parfaites qu'elles nous semblent refusées en ce monde? Cette dernière objection présuppose que, pour être authentique, il faudrait commencer par se connaître. Mais ne se repose-t-elle pas sur une conception trop «réfléchie » et intellectualiste de ce que signifie en réalité l'authenticité? Pour être authentique, je n'ai pas besoin de vouloir l'être, de viser cette qualité comme un but, ni de savoir que je le suis au moment où je le suis. Comme l'ont montré abondamment les conceptions du naturel, de Montaigne aux Moralistes classiques, la recherche délibérée du naturel et le soin mis à l'obtenir contreviennent à leur propre objectif. L'authenticité n'a nul besoin de devenir un but conscient et réfléchi, ni le but de nos efforts, pour être possible; à son niveau le plus simple, elle réside simplement dans une absence de dissimulation, de fraude, de tricherie, de même que la sincérité consiste uniquement dans une abstention de tout mensonge. Qui plus est, comme l'ont observé les penseurs du naturel, la recherche délibérée de l'authenticité conduit souvent à l'opposé de cette dernière. Que l'on songe à Rousseau: sa revendication incessante de sa propre sincérité finit par rendre celle-ci douteuse aux yeux du lecteur, dans la mesure où elle emploie en réalité le même genre de subterfuge que celui dont use le menteur: celui-ci, pour mentir avec succès, doit faire croire à autrui ce qu'il affirme; de même, l'authentique qui se veut tel et se revendique tel doit chercher à faire croire à autrui qu'il l'est, et ainsi, souvent, se le faire croire à luimême; or il y a trop de recherche d'effets dans une telle attitude pour ne pas rendre douteux l'objet même de sa revendication.

Il y a des cas où l'excès de connaissance de soi et de réflexion détruit la possibilité même de l'authenticité. Supposons un homme qui vient de rencontrer une femme pour laquelle il commence à éprouver des sentiments; 
elle l'invite à la rejoindre au bord de la mer, et il en ressent un vif désir; mais en même temps, il a d'autres obligations: il aspire à une promotion importante et il sait que s'il laisse tout en plan pour la rejoindre, cette promotion lui sera refusée. Alors que fait-il ? Il se met à réfléchir, à se sonder, à s'introspecter, à se demander ce qui est le plus important pour lui entre rejoindre cette quasi-inconnue pour laquelle il éprouve ces sentiments naissants ou donner la priorité à sa carrière. Supposons qu'à l'issue de cette longue délibération, après avoir pesé le pour et le contre, il aboutisse à la conclusion qu'il ferait bien de rejoindre cette femme. Ici, sa réflexion, manifestement, est une réflexion de trop. Car même s'il décide d'accepter l'invitation, il rejoint cette femme pour des motifs fondamentalement égoïstes, et non par amour. Il a eu ce que Bernard Williams appelle joliment "une pensée de trop ». La conduite authentique, ici, aurait été celle dans laquelle il se serait abstenu de peser le pour et le contre et aurait simplement cédé à ses sentiments - quelles qu'en eussent été les conséquences du point de vue de sa carrière. Il y a des choses que l'on fait par amour, et y ajouter un motif additionnel, c'est y ajouter un motif qui contredit cette disposition. Ainsi, non seulement l'authenticité s'accommode parfois de l'absence de réflexion, mais la réflexion, dans certaines circonstances au moins, contredit la possibilité de l'authenticité ${ }^{40}$. Il ne faut pas se faire une conception trop réflexive de cette qualité existentielle.

40. On pourra se reporter aux remarques de Simon Feldman et Allan Hazlett, "Authenticity and Self-Knowledge", Dialectica, vol. 67, n² 2, p. I 57-I 8I. 